OPEN ACCESS

Edited by:

Paola Marziani,

Osservatorio Astronomico di Padova

(INAF), Italy

Reviewed by:

Alenka Negrete,

Universidad Nacional Autónoma de

México, Mexico

Dawei Xu,

National Astronomical Observatories

(CAS), China

${ }^{*}$ Correspondence:

Paolo Padovani

ppadovan@eso.org

Specialty section

This article was submitted to Milky Way and Galaxies,

a section of the journal

Frontiers in Astronomy and Space

Sciences

Received: 14 September 2017

Accepted: 23 October 2017

Published: 07 November 2017

Citation:

Padovani P (2017) Active Galactic Nuclei at All Wavelengths and from All

Angles.

Front. Astron. Space Sci. 4:35.

doi: 10.3389/fspas.2017.00035

\section{Active Galactic Nuclei at All Wavelengths and from All Angles}

\author{
Paolo Padovani * \\ European Southern Observatory, Garching bei München, Germany
}

AGN are quite unique astronomical sources emitting over more than 20 orders of magnitude in frequency, with different electromagnetic bands providing windows on different sub-structures and their physics. They come in a large number of flavors only partially related to intrinsic differences. I highlight here the types of sources selected in different bands, the relevant selection effects and biases, and the underlying physical processes. I then look at the "big picture" by describing the most important parameters one needs to describe the variety of AGN classes and by discussing AGN at all frequencies in terms of their sky surface density. I conclude with a look at the most pressing open issues and the main new facilities, which will flood us with new data to tackle them.

Keywords: quasars, active galactic nuclei, supermassive black holes, radio emission, infrared emission, $\mathrm{X}$-ray emission, $\gamma$-ray emission

\section{INTRODUCTION}

AGN are, by definition, more powerful than the nuclei of normal galaxies. This "additional" component is now universally accepted to be due to an actively accreting central supermassive ( $10^{6} M_{\odot}$ ) black hole (SMBH). This implies a number of fascinating properties, which include very high luminosities (up to $L_{\mathrm{bol}} \approx 10^{48} \mathrm{erg} \mathrm{s}^{-1}$ ), small emitting regions in most bands (of the order of a milliparsec), strong evolution of the luminosity functions, and broad-band emission covering the entire electromagnetic spectrum (see Padovani et al., 2017, for a review).

The latter property means that AGN have been discovered at all wavelengths. This is partly responsible for the very large number of classes and sub-classes AGN researchers have come up with (see Table 1), which appear to be overwhelming and very confusing, especially to astronomers working in other fields and particularly to physicists.

Different methods are employed to select AGN in the various bands, which also provide different views on AGN physics. I discuss this next. I refer to Padovani et al. (2017) for a more in-depth review of these (and other) topics.

\section{THE RADIO BAND}

One of the main results of the past few years has been the realization that the radio sky population undergoes major changes at low flux densities. Namely, while the bright radio sky $\left(f_{\mathrm{r}} \gtrsim 1 \mathrm{mJy}\right.$ around $1 \mathrm{GHz}$, where $1 \mathrm{Jy}$ is $10^{-23} \mathrm{erg} \mathrm{cm}^{-2} \mathrm{~s}^{-1} \mathrm{~Hz}^{-1}$ ) is populated mostly by radio galaxies (RGs) and radio quasars, that is largely non-thermal sources, at faint radio flux densities we are now detecting mainly star-forming galaxies (SFGs) and the more common non-jetted AGN (see Padovani, 2016, for a review). This change is also apparent by looking at the Euclidean normalized $1.4 \mathrm{GHz}$ source counts, which show an upturn around $\approx 0.1 \mathrm{mJy}$ (see Figure 1 ). We therefore need to deal separately with the two flux density regimes. 
TABLE 1 | The AGN zoo: list of AGN classes.

\begin{tabular}{|c|c|}
\hline Class/Acronym & Meaning/Main properties \\
\hline Quasar & Quasi-stellar radio source (originally) \\
\hline Sey1 & Seyfert $1, \mathrm{FWHM} \gtrsim 1,000 \mathrm{~km} \mathrm{~s}^{-1}$ \\
\hline Sey2 & Seyfert $2, \mathrm{FWHM} \lesssim 1,000 \mathrm{~km} \mathrm{~s}^{-1}$ \\
\hline QSO & Quasi-stellar object \\
\hline QSO2 & Quasi-stellar object 2, high power Sey2 \\
\hline RQ AGN & Radio-quiet AGN \\
\hline RL AGN & Radio-loud AGN \\
\hline Jetted AGN & With strong relativistic jets \\
\hline Non-jetted AGN & Without strong relativistic jets \\
\hline Type 1 & Sey1 and quasars \\
\hline Type 2 & Sey2 and QSO2 \\
\hline FRI & Fanaroff-Riley class I radio source \\
\hline FR ॥ & Fanaroff-Riley class II radio source \\
\hline BL Lac & BL Lacertae object \\
\hline Blazars & BL Lacs and FSRQs \\
\hline $\mathrm{BAL}$ & Broad absorption line (quasar) \\
\hline $\mathrm{BLO}$ & Broad-line object, FWHM $\gtrsim 1,000 \mathrm{~km} \mathrm{~s}^{-1}$ \\
\hline BLAGN & Broad-line AGN, FWHM $\gtrsim 1,000 \mathrm{~km} \mathrm{~s}^{-1}$ \\
\hline BLRG & Broad-line radio galaxy \\
\hline $\mathrm{CDQ}$ & Core-dominated quasar \\
\hline CSS & Compact steep spectrum radio source \\
\hline CT & Compton-thick \\
\hline FR 0 & Fanaroff-Riley class 0 radio source \\
\hline FSRQ & Flat-spectrum radio quasar \\
\hline GPS & Gigahertz-peaked radio source \\
\hline $\mathrm{HBL} / \mathrm{HSP}$ & High-energy cutoff BL Lac/blazar \\
\hline HEG & High-excitation galaxy \\
\hline $\mathrm{HPQ}$ & High polarization quasar \\
\hline \multicolumn{2}{|l|}{ Jet-mode } \\
\hline IBL/ISP & Intermediate-energy cutoff BL Lac/blazar \\
\hline LINER & Low-ionization nuclear emission-line regions \\
\hline LLAGN & Low-luminosity AGN \\
\hline LBL/LSP & Low-energy cutoff BL Lac/blazar \\
\hline LDQ & Lobe-dominated quasar \\
\hline LEG & Low-excitation galaxy \\
\hline $\mathrm{LPQ}$ & Low polarization quasar \\
\hline NLAGN & Narrow-line AGN, FWHM $\lesssim 1,000 \mathrm{~km} \mathrm{~s}^{-1}$ \\
\hline NLRG & Narrow-line radio galaxy \\
\hline NLS1 & Narrow-line Seyfert 1 \\
\hline OW & Optically violently variable (quasar) \\
\hline \multicolumn{2}{|l|}{ Population A } \\
\hline \multicolumn{2}{|l|}{ Population B } \\
\hline \multicolumn{2}{|l|}{ Radiative-mode } \\
\hline $\mathrm{RBL}$ & Radio-selected BL Lac \\
\hline Sey 1.5 & Seyfert 1.5 \\
\hline Sey 1.8 & Seyfert 1.8 \\
\hline Sey1.9 & Seyfert 1.9 \\
\hline SSRQ & Steep-spectrum radio quasar \\
\hline USS & Ultra-steep spectrum source \\
\hline $\mathrm{XBL}$ & X-ray-selected BL Lac \\
\hline XBONG & X-ray bright optically normal galaxy \\
\hline
\end{tabular}

The top part of the table relates to major/classical classes. See Padovani et al. (2017) for a more complete version of this table, including more details and references.

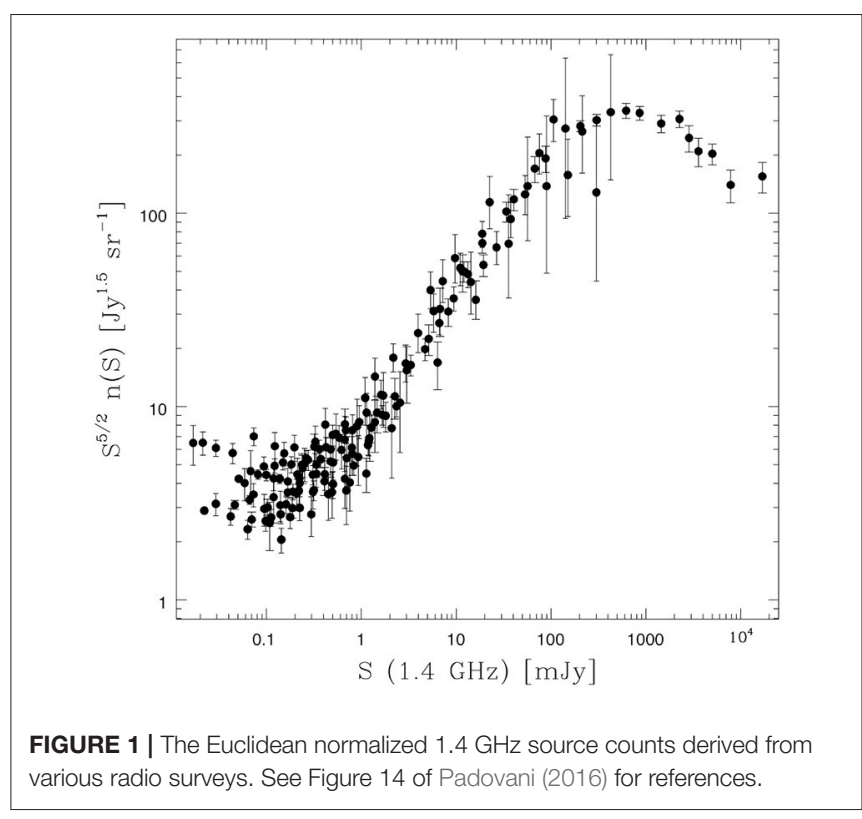

\subsection{The Bright Radio Sky: $\boldsymbol{f}_{\mathrm{r}} \gtrsim \mathbf{1} \mathrm{mJy}$}

Sources include mostly jetted AGN, mainly blazars and RGs. Selection is done by just observing the high Galactic latitude sky as AGN are (basically) the only sources. This is helped by the fact that stars are extremely weak radio emitters. For example, out of the 527 sources with $5 \mathrm{GHz}$ flux density $>1 \mathrm{Jy}$ and Galactic latitude $\left|b_{I I}\right| \geq 10^{\circ}$ (Kühr et al., 1981) only M 82 is not an AGN (and only M 82 and NGC 1068 do not belong to the RG, radio quasar, or blazar classes; moreover, $\sim 51 \%$ of the classified sources are blazars). The radio band probes the jet and its emission is due to synchrotron radiation (ultra-relativistic electrons in a magnetic field). The only bias is that we are sampling the jetted AGN population, which is only a small fraction $(<10 \%)$ of the total.

\subsection{The Faint Radio Sky: $\boldsymbol{t}_{\mathrm{r}} \lesssim 1 \mathrm{mJy}$}

Sources include both non-jetted AGN and a quickly decreasing fraction of jetted AGN (see Figure 7 of Padovani, 2016); the former are the dominant type. Selection requires data in various bands to single out the AGN, especially the non-jetted ones, from the SFGs, as the optical counterparts are very faint (as detailed in section 5.1 of Padovani, 2016). Apart from probing the jet in jetted AGN, radio emission is mostly due to star formation (through synchrotron emission from relativistic plasma accelerated in supernova remnants) in non-jetted ones (at least for radio-selected sources: see discussion in section 6.2 of Padovani, 2016). There is some chance of contamination from SFGs (especially if no X-ray detection is available). But we are now reaching the most common non-jetted AGN with no obscuration bias.

I would also like to stress, once more (see also Padovani, 2016, 2017), that the differences between jetted and non-jetted AGN are not taxonomic but reflect intrinsic differences, with jetted AGN emitting a large fraction of their energy in association 


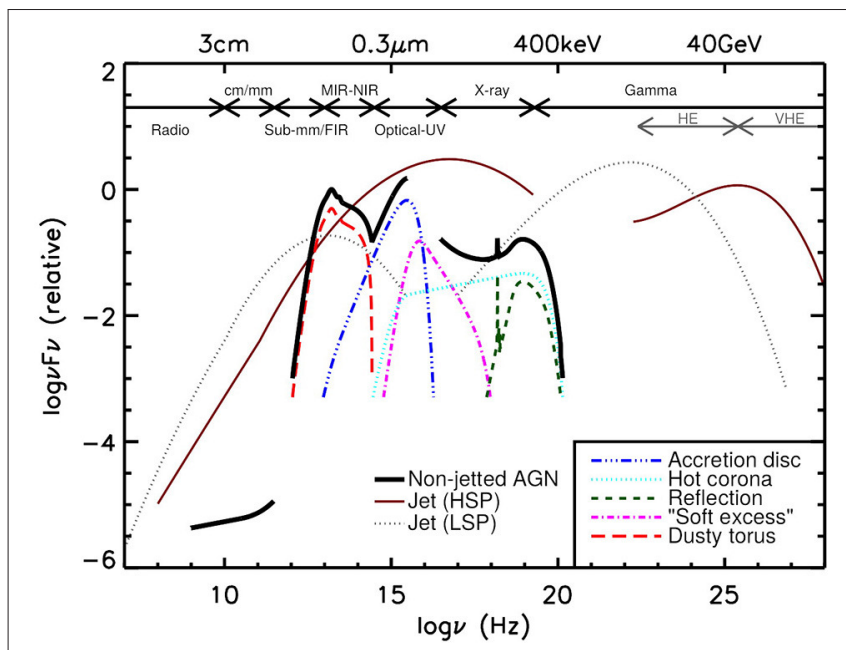

FIGURE 2 | A schematic representation of an AGN spectral energy distribution (SED), loosely based on the observed SEDs of non-jetted quasars (e.g., Elvis et al., 1994; Richards et al., 2006). The black solid curve represents the total emission and the various colored curves (shifted down for clarity) represent the individual components. The intrinsic shape of the SED in the $\mathrm{mm}$-far infrared (FIR) regime is uncertain; however, it is widely believed to have a minimal contribution (to an overall galaxy SED) compared to star formation (SF), except in the most intrinsically luminous quasars and powerful jetted AGN. The primary emission from the AGN accretion disk peaks in the UV region. The jet SED is also shown for a high synchrotron peaked blazar (HSP, based on the SED of Mrk 421) and a low synchrotron peaked blazar (LSP, based on the SED of 3C 454.3). Adapted from Harrison (2014). Image credit: C. M. Harrison. Figure reproduced from Padovani et al. (2017), Figure 1, with permission of Springer.

with powerful relativistic jets and therefore non-thermally, while the multi-wavelength emission of non-jetted AGN is dominated by thermal emission related to the accretion disk, which forms around the SMBH. Because of this, only jetted AGN manage to emit in the hard X-ray to $\gamma$-ray bands (see also Figure 2). Furthermore, the 50-year-old "radio-loud/radio-quiet" labels are obsolete, misleading, and wrong and should be dropped (Padovani, 2016, 2017).

\section{THE INFRARED BAND}

Whatever its detailed distribution and properties, we know there is dust around most AGN outside the accretion disk and on scales larger than those of the broad line region (BLR). This dust has a temperature in the $100-1,000 \mathrm{~K}$ range and is located at a minimum distance determined by the sublimation temperature of the dust grains (Barvainis, 1987). Ultraviolet (UV)/optical emission from the accretion disk is absorbed by it and re-emitted in the infrared (IR) band where it dominates the AGN spectral energy distribution (SED) at $\lambda \gtrsim 1 \mu \mathrm{m}$ up to a few tens of micron (Figure 2).

Dust plays a vital role in AGN unification schemes (e.g., Antonucci, 1993; Urry and Padovani, 1995). Thanks to polarization studies it was in fact realized that the difference between type 1 (broad-lined) and type 2 (narrow-lined) AGN was solely due to orientation with respect to the dust. In the latter objects our view of the accretion disk and the BLR is obscured by the dust and therefore we cannot see the UV bump and the broad lines typical of type 1 AGN but can only detect the narrow lines emitted by slower-moving clouds beyond the dust (but see Elitzur and Netzer, 2016 for a discussion about possible real type 2 AGN where the difference is not caused by dust obscuration; see also section 5.3 of Padovani et al., 2017).

Importantly, it now looks like the dust and the BLR are only present at high powers $\left(\gtrsim 10^{42} \mathrm{erg} \mathrm{s}^{-1}\right) /$ high Eddington ratios $\left(L / L_{\text {Edd }} \gtrsim 0.01\right.$; see discussion in Padovani et al., 2017), which implies that dust-driven unification breaks down below these values. Jet-driven, low-power unification (linking BL Lacs with LEG RGs) is unaffected by this.

Based on all of the above, AGN selected in the IR band include mostly non-jetted AGN (because low-power/low accretion rate jetted sources do not have any dust) of the radiative-mode type (i.e., with high accretion rates and $L / L_{\mathrm{Edd}}$ ). The IR is sensitive to both obscured and unobscured AGN, providing an almost isotropic selection, and in particular to extremely obscured AGN (missed by optical and soft X-ray surveys). Selection is done by typically using IR colors with the aim of separating AGN from SFGs (see section 3.2 in Padovani et al., 2017, and specifically Figure 5 and Table 2). While for shallow surveys relatively high reliability and completeness can be obtained (see caption of Table 2 for definitions) this is not the case for deeper ones, where high reliability can only be obtained by having low completeness (in other words, one can select mostly AGN only by missing a large fraction of them above a given flux limit).

\section{THE OPTICAL/UV BAND}

Optical/UV emission in AGN comes from the accretion disk and the BLR. Because of the presence of dust discussed in section 3 one can detect this emission only if our line of sight is unobstructed (which happens only for specific orientations) and therefore only in unobscured sources. The optical/UV band, therefore, provides a very biased view of the AGN phenomenon, although it was also thanks to their strong optical/UV emission that AGN were mostly discovered in the past.

AGN selected in the optical/UV band, therefore, include unobscured sources mostly of the non-jetted type [as only a small fraction of jetted AGN (radio quasars) have also a standard accretion disk and a BLR]; in short, type 1 AGN. Predictably, this band misses lots of obscured AGN (the type 2 s), although many of them are still selected through their narrow emission lines, and even the moderately obscured ones. Other biases are against low-luminosity AGN (where the host galaxy light swamps the AGN) and also AGN close to stellar loci (as stars are also strong optical/UV emitters) especially at $z \sim 2.6$ and 3.5.

The optical/UV band, however, makes up for these shortcomings in two ways: (1) by providing spectra which are vital to study AGN physics, e.g., the accretion disk, to estimate the mass of the central SMBH through "reverberation mapping", and to study the AGN spectral diversity; (2) by supplying us with huge optical catalogs. More details on these topics can be found in section 4 of Padovani et al. (2017). 
TABLE 2 | A multi-wavelength overview of AGN highlighting the different selection biases (weaknesses) and key capabilities (strengths).

\begin{tabular}{|c|c|c|c|c|}
\hline Band & Type & Physics & Selection biases/weaknesses & Key capabilities/strengths \\
\hline Radio, $f_{r} \gtrsim 1 \mathrm{mJy}$ & Jetted & Jet & Non-jetted sources & $\begin{array}{l}\text { High efficiency, } \\
\text { no obscuration bias }\end{array}$ \\
\hline Radio, $f_{\mathrm{r}} \lesssim 1 \mathrm{mJy}$ & Jetted and non-jetted & Jet and SF & Host contamination & $\begin{array}{l}\text { Completeness, } \\
\text { no obscuration bias }\end{array}$ \\
\hline IR & Type 1 and 2 & $\begin{array}{l}\text { Hot dust } \\
\text { and SF }\end{array}$ & $\begin{array}{l}\text { Completeness, reliability, } \\
\text { host contamination, no dust }\end{array}$ & $\begin{array}{l}\text { Weak obscuration bias, } \\
\text { high efficiency }\end{array}$ \\
\hline Optical & Type 1 & Disk & $\begin{array}{l}\text { Completeness, low-luminosity, } \\
\text { obscured sources, host contamination }\end{array}$ & $\begin{array}{l}\text { High efficiency, } \\
\text { detailed physics from lines }\end{array}$ \\
\hline X-ray & Type 1 and (most) 2 & Corona & $\begin{array}{l}\text { Very low-luminosity, } \\
\text { heavy obscuration }\end{array}$ & $\begin{array}{l}\text { Completeness, low } \\
\text { host contamination }\end{array}$ \\
\hline$\gamma$-ray & Jetted & Jet & $\begin{array}{l}\text { Non-jetted, } \\
\text { unbeamed sources }\end{array}$ & High reliability \\
\hline Variability & All (in principle) & $\begin{array}{l}\text { Corona, } \\
\text { disk, jet }\end{array}$ & $\begin{array}{l}\text { Host contamination, obscuration, } \\
\text { cadence and depth of observations }\end{array}$ & Low-luminosity \\
\hline
\end{tabular}

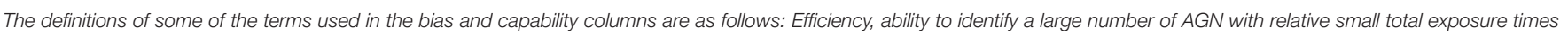

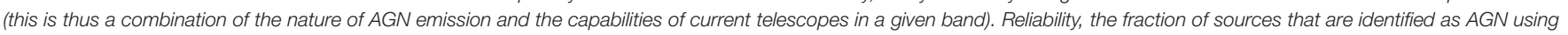

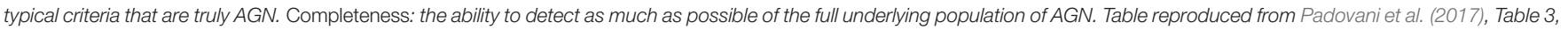
with permission of Springer.

\section{THE X-RAY BAND}

$\mathrm{X}$-ray emission appears to be one of the defining features of AGN and therefore the X-ray band has proven to be very important for AGN studies. X-rays are deemed to be due to inverse Compton scattering of the accretion-disk photons by an atmosphere above the accretion disk (referred to as the "corona" and whose geometry is still unknown). In jetted AGN the X-rays can have a major contribution from the jet as well. The $\mathrm{X}$ rays then interact with matter by being reflected, scattered, and absorbed by the accretion disk and/or the dust discussed in section 3. X-ray spectra are sensitive to all of these components and, in particular, to the amount of absorbing material, which means they can be also used to classify sources into absorbed (type 2) and unabsorbed (type 1). This is because lower energy $\mathrm{X}$-rays are more easily absorbed than higher energy ones and so the shape of the spectrum depends on the column density $N_{\mathrm{H}}$. When $N_{\mathrm{H}}>1.5 \times 10^{24} \mathrm{~cm}^{-2}$ (in so-called Compton-thick [CT] sources) the source looks completely absorbed in the X-ray band.

$\mathrm{X}$-ray selected AGN, then, include in theory all AGN. However, sources with progressively larger $N_{\mathrm{H}}$ will be systematically missed below an increasingly higher energy, until the CT value is reached when all AGN are undetectable in the X-ray band. Low-luminosity AGN with $L_{\mathrm{x}} \lesssim 10^{42} \mathrm{erg} \mathrm{s}^{-1}$ are also biased against as this is the power associated with host galaxy emission. For the same reason at very low soft X-ray fluxes $\left(f_{0.5-2 \mathrm{keV}} \lesssim 6 \times 10^{-18} \mathrm{erg} \mathrm{cm}^{-2} \mathrm{~s}^{-1}\right)$ contamination from SFGs kicks in. Low-power RGs (i.e., of the LEG type) are also mostly missed since their X-ray jet emission is not very strong and they lack an accretion disk. More details on these topics can be found in section 5 of Padovani et al. (2017).

\section{THE $\gamma$-RAY BAND}

The $\gamma$-ray band is conventionally divided into the High Energy (HE) band, between $100 \mathrm{MeV}\left(2.4 \times 10^{22} \mathrm{~Hz}\right)$ and $\sim 100 \mathrm{GeV}$ $\left(2.4 \times 10^{25} \mathrm{~Hz}\right)$, and the Very High Energy (VHE) band, covering the $\sim 50 \mathrm{GeV}$ to $\sim 10 \mathrm{TeV}\left(2.4 \times 10^{27} \mathrm{~Hz}\right)$ range. Different detectors operate in the two bands: in the former we have electron pair-conversion telescopes in space characterized by a very large field of view (FoV; thousands of square degrees) (e.g., AGILE and Fermi: Atwood et al., 2009; Tavani et al., 2009); VHE $\gamma$-rays, on the other hand, are detected by Imaging Atmospheric Cherenkov Telescopes and Extensive Air Shower observatories on the ground ${ }^{1}$ (e.g., Cangaroo, Hess, Magic, Veritas, and HAWC: de Naurois and Mazin, 2015; Abeysekara et al., 2017).

$\gamma$-ray astronomy is still a relatively young branch so its number of sources is much smaller compared to the other bands. The Third Fermi source catalog (Acero et al., 2015) has detected 3,033 sources all-sky above $100 \mathrm{MeV}$ and up to $300 \mathrm{GeV}$. AGN make up $\approx 60 \%$, and possibly $\lesssim 90 \%$ (including the still unclassified sources), of these. These include only jetted AGN, the large majority of them being blazars with only a handful of RGs. Therefore, the current $\gamma$-ray sky is quite similar to the radiobright sky, which is also dominated by blazars (section 2.1). This is simply because both radio and $\gamma$-ray photons are sampling jet-related, non-thermal processes.

\footnotetext{
${ }^{1}$ These instruments observe at ground level the particle showers that are produced by the interaction of VHE $\gamma$-ray photons with the top layers of the atmosphere through the Cherenkov light they create or via the detection of the charged particles in the shower.
} 
The VHE sky is even more sparsely populated, as only $\sim 200$ sources have been detected at $\mathrm{TeV}$ energies ${ }^{2}$. Of those $\sim 35 \%$ are AGN, mostly blazars. Blazars, so far, make up $\sim 92 \%$ of the extragalactic $\gamma$-ray sky, with the remaining fraction including LEG RGs $(\sim 6 \%)$ and SFGs $(\sim 2 \%)$.

$\gamma$-ray selected AGN, therefore, include only jetted AGN, mostly blazars. There is then an obvious bias against non-jetted AGN, which is simply due, in my opinion, to the fact that these sources lack the engine to produce $\gamma$-ray photons (although they could still be very weak $\gamma$-ray sources through proton-proton collisions [via $p p \rightarrow \pi^{0} \rightarrow 2 \gamma$ : the so-called "pion decay"] thanks to their outflows: Wang and Loeb, 2016; Lamastra et al., 2017).

Although it is clear that $\gamma$-rays are produced by relativistic jets, the exact process through which this happens is still not understood. Most researchers favor leptonic processes, where the electrons in the jet inverse Compton scatter their own synchrotron photons (synchrotron self-Compton) or an external photon field (external inverse Compton). In hadronic scenarios, on the other hand, $\gamma$-rays are instead thought to stem from high-energy protons either loosing energy through synchrotron emission or through pion decay. In the hadronic case blazars would also produce neutrinos (from charged pions decay) and possibly also cosmic rays becoming therefore multi-messenger sources (e.g., Padovani et al., 2016; Resconi et al., 2017).

\section{PUTTING IT ALL TOGETHER}

\subsection{Making Order Out of Chaos: A Minimalistic Approach to AGN}

Figure 2 brings home one of the main points of this paper: different AGN components emit in different bands and only by looking at the broad, multi-wavelength picture can we understand AGN. But different bands gives us also different perspectives and types of sources, which means we need to be fully aware of selection effects. The selection biases and key capabilities (i.e., the weaknesses and the strengths) of all bands discussed above are summarized in Table 2.

Based on our current understanding of AGN we can now look back at Table $\mathbf{1}$ and see that the complexity it displays is only apparent. It has been known for quite some time that many of the different classes can be explained by taking orientation and the presence (or lack) of a relativistic jet into account. More recent evidence suggests that other parameters play a role, above all the accretion rate (or equivalently $L / L_{\text {Edd }}$ see section 8.1 of Padovani et al., 2017 for references). This can change the structure of the accretion flow and thus the SED shape. I am convinced that these three parameters (i.e., orientation, relativistic jet, and accretion rate) explain most of the AGN variety. For example, BL Lacs are LEG RGs with their jets pointing toward the observer and also the radiatively inefficient version (with lower accretion rate and $L / L_{\mathrm{Edd}}$ ) of FSRQs. Additionally, possible second-order parameters include the galaxy environment (e.g., Chiaberge et al., 2015) and the host galaxy, which can also partially obscure the AGN (e.g., Buchner and Bauer, 2017).

${ }^{2}$ http://tevcat.uchicago.edu/

\subsection{AGN at All Frequencies}

AGN emit over the full electromagnetic spectrum (and possibly even beyond it). The ease with which AGN are detected in the various bands, however, depends essentially on three aspects: (1) astrophysics. As shown by Figure 2 AGN do not emit at the same level in all bands and the SED is shaped by the various AGN components. The most manifest of these is the presence or absence of a strong relativistic jet, which leads to the distinction between jetted and non-jetted AGN but also modifies the SED substantially (the latter class is weaker in the radio and not present in the $\gamma$-ray band). Other effects include changes in the accretion rate (or $L / L_{\mathrm{Edd}}$ ), which can also modify the SED predominantly in the optical/UV and X-ray bands, and obviously obscuration by gas and dust that can absorb emission from the near-IR (NIR) to the soft X-rays; (2) selection effects. AGN selection techniques are not uniformly efficient in the various bands, resulting in (sometimes strong) biases. For example, the host galaxy in the optical, IR, and to some extent the radio band can be particularly luminous making the selection of faint AGN more challenging; host contamination is instead particularly low in the X-ray band; (3) technology. Not all astronomical detectors are equally efficient (it is much harder to catch a high-energy photon than a low-energy one) which means that observations in some wavebands are going to be more sensitive than others relative to the typical AGN SED. For example, contemporary radio telescopes can detect jetted AGN with much smaller bolometric fluxes compared to current $\gamma$-ray observatories.

This complex mix of physics, selection effects, and technology is behind the numbers in Figure 3, which shows the largest AGN surface density over the whole electromagnetic spectrum. Thanks to cutting-edge $\mathrm{X}$-ray observatories and the low contamination from the host galaxy the AGN surface density is largest in the soft X-ray band. And the difficulty of detecting $\gamma$-ray photons combined with the fact that only a minority of AGN (the jetted ones) are $\gamma$-ray emitters result in a very low surface density in this band. Furthermore, despite being intrinsically radio faint, non-jetted AGN have a surface density in the radio band larger than that reached by the deepest optical surveys and at the same level as the NIR values, which highlights the sensitivity reached by present radio facilities. Since radio observations are unaffected by absorption they are also sensitive to all types of AGN, irrespective of their orientation (i.e., type $1 \mathrm{~s}$ and type $2 \mathrm{~s}$ ), unlike soft X-ray ones.

Three more considerations (of the many more one could make!) are in order about Figure 3: (1) the actual number of detected and identified AGN has a wavelength dependence very different from that shown in the figure and is still heavily biased toward the optical/NIR bands, as most AGN were discovered through dedicated large-area spectroscopic surveys. The figure could then be thought of as describing the "detection potential" of the various bands; (2) the largest entry in the figure translates into at least $\approx 1$ billion AGN in the Universe that could be detected with current technology. The comparison with the number of currently known AGN (of the order of a million: see Figure 6 of Padovani et al., 2017) shows that there is still a lot of room for discovery in future AGN surveys (see section 8); (3) it is worth reflecting on the large financial and human investment behind 


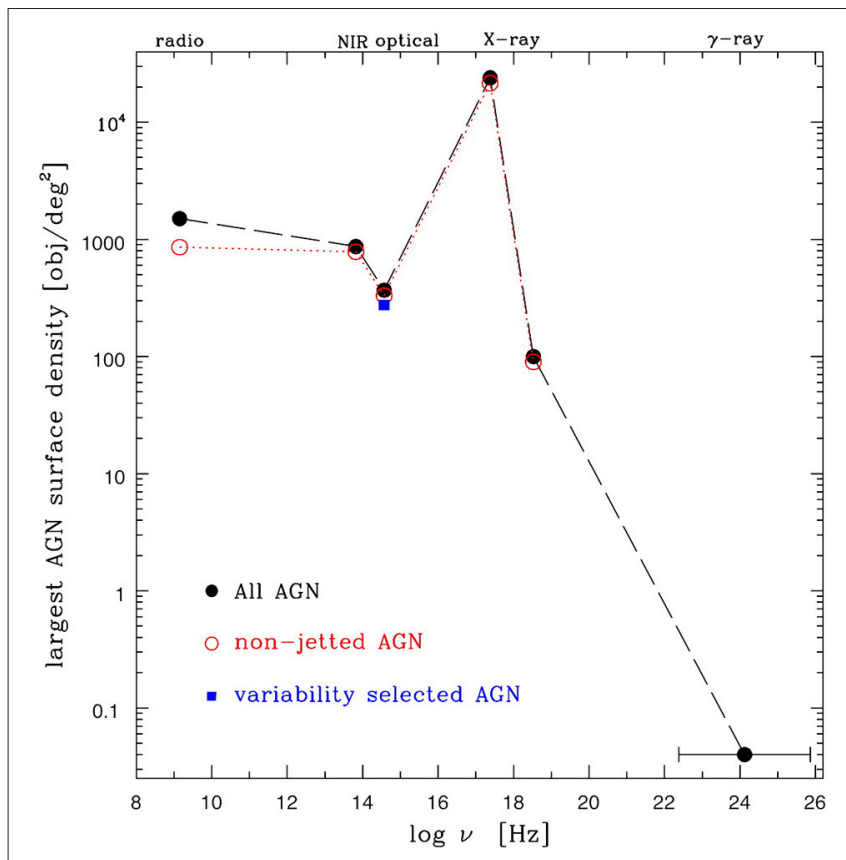

FIGURE 3 | The largest AGN surface density over the whole electromagnetic spectrum. Black filled points refer to all AGN, open red points are for non-jetted AGN. The latter are actually directly measured only in the radio band, while in the NIR to X-ray bands they have been derived by simply subtracting $10 \%$ from the total values. Non-jetted AGN have not been detected in the $\gamma$-ray band. The blue square indicates variability selected AGN. Updated from Figure 11 of Padovani (2016), where one can find the references to the relevant samples and facilities, to include variability selected $A G N$ and the results of the CDFS $7 \mathrm{Ms}$ sample (Luo et al., 2017). Figure reproduced from Padovani et al. (2017), Figure 21, with permission of Springer.

the figure, which could only be put together thanks to state-ofthe-art ground- and space-based facilities (the ones listed in the caption of Figure 11 of Padovani, 2016 do not take into account the multi-wavelength data most of the surveys had to accumulate in order to be able to identify their targets) and the effort of many teams.

\section{OPEN ISSUES AND THE NEAR FUTURE}

We have learnt a lot about AGN since the discovery of the first quasar in 1963. However, there are still many open questions in AGN research, some of them quite important, to say the least, a comprehensive list of which is given in section 8.4 of Padovani et al. (2017). My favorite topics include:

- Why do a minority of AGN have jets? There are some hints, as jetted AGN appear to be more clustered, undergo mergers, reside in more massive, bulge-dominated galaxies (and perhaps spin faster) than non-jetted AGN. But the truth is this is still an unsolved issue.

- What is the composition, geometry, and morphology of the obscuring dust? Recent ground-based mid-IR interferometric observations have suggested that our "standard" (doughnutlike) picture of the so-called dusty torus might not be always valid and that a large fraction of the dust might instead reside in the walls of the ionization cone (e.g., López-Gonzaga et al., 2016, and references therein).

- How does the cosmic history of black hole accretion as traced by AGN compare to the history of star formation in galaxies? More generally, what is the physical connection between $\mathrm{SMBH}$ evolution and that of their host galaxies and halos, and are these driven by common processes? This is a very hot topic, which has attracted a lot of attention in the past few years and has important implications for galaxy evolution. Despite this, we still do not have definite answers to these questions.

- Are blazars multi-messenger emitters? Said otherwise, is there a connection between $\gamma$-ray emission from jetted AGN, neutrinos and cosmic rays? If so, this would have huge implications for astro-particle physics, solving at the same time the mystery of the origin of (at least some of) the IceCube neutrinos and ultra high-energy cosmic rays detected, amongst others, by the Pierre Auger Observatory and the Telescope Array. It would also constrain theoretical models for particle acceleration in AGN by proving beyond any doubt the existence of very energetic protons.

We will soon have plenty of data to tackle these and other open issues. Here I simply list some of the main future facilities, which we will be having at our disposal in the next few years, sorted by band. Many more details and relevant hyperlinks can be found in Padovani et al. (2017).

- Radio: ASKAP (Australia), MeerKAT (South Africa), eMERLIN (UK), APERTIF (The Netherlands), and finally the Square Kilometre Array;

- IR: JWST (NASA/ESA), Tokyo Atacama Observatory (Japan), Euclid (ESA/NASA), WFIRST (NASA), SPICA;

- Optical/NIR: Zwicky Transient Facility (USA), LSST, and the giant telescopes namely GMT, TMT, and the ELT;

- X-ray: eROSITA (Germany/Russia), XIPE, and Athena;

- $\gamma$-ray: Cherenkov Telescope Array and the Large High Altitude Air Shower Observatory (China).

In terms of AGN science these facilities will open up entire new regions of parameter space, especially with regard to sensitivity and number of sources. Just to give a few numbers: the Evolutionary Map of the Universe, one of the ASKAP surveys, will detect approximately 30 million AGN in the radio band; Euclid will provide NIR spectra for $\approx 1$ million AGN; the LSST will select more than 10 million AGN; eROSITA will provide $\mathrm{X}$-ray data for $\approx 3$ million AGN; and the Cherenkov Telescope Array will detect $\sim 10$ times more blazars than are currently known at $\mathrm{TeV}$ energies.

In short, the future of AGN studies is very bright and we will soon be flooded with amazing new data. To take full advantage of them we need to ask the right questions and use the most appropriate and efficient tools.

\section{MAIN MESSAGES}

The main points of this paper can be thus summarized:

1. Different bands give us different perspectives, different physics, and different types of AGN: one needs to be aware of selection effects! 
2. The "AGN zoo" is unnecessarily complex: most of the variety of AGN classes can be explained by three main parameters, namely orientation, presence or lack of relativistic jets, and accretion rate. Second-order parameters might include the environment and the host galaxy.

3. There are two main classes of AGN: jetted and non-jetted (not "radio-loud" and "radio-quiet")!

4. In the next few years we will be flooded with AGN data. We need to be ready for them by: (1) asking the right questions; (2) having the right tools to analyze them.

\section{REFERENCES}

Abeysekara, A. U., Albert, A., Alfaro, R., Alvarez, C., Álvarez, J. D., Arceo, R., et al. (2017). The 2 HWC HAWC observatory gamma-ray catalog. Astrophys. J. 843:40. doi: $10.3847 / 1538-4357 /$ aa7556

Acero, F., Ackermann, M., Ajello, M., Albert, A., Atwood, W. B., Axelsson, M., et al. (2015). Fermi large area telescope third source catalog. Astrophys. J. Suppl. 218:23. doi: 10.1088/0067-0049/218/2/23

Antonucci, R. (1993). Unified models for active galactic nuclei and quasars. Ann. Rev. Astron. Astrophys. 31, 473-521. doi: 10.1146/annurev.aa.31.090193.002353

Atwood, W. B., Abdo, A. A., Ackermann, M., Althouse, W., Anderson, B., Axelsson, M., et al. (2009). The large area telescope on the fermi gamma-ray space telescope mission. Astrophys. J. 697, 1071-1102. doi: 10.1088/0004-637X/697/2/1071

Barvainis, R. (1987). Hot dust and the near-infrared bump in the continuum spectra of quasars and active galactic nuclei. Astrophys. J. 320, 537-544. doi: $10.1086 / 165571$

Buchner, J., and Bauer, F. E. (2017). Galaxy gas as obscurer - II. Separating the galaxy-scale and nuclear obscurers of active galactic nuclei. Mon. Not. R. Astron. Soc. 465, 4348-4362. doi: 10.1093/mnras/stw2955

Chiaberge, M., Gilli, R., Lotz, J. M., and Norman, C. (2015). Radio loud AGNs are mergers. Astrophys. J. 806:147. doi: 10.1088/0004-637X/806/2/147

de Naurois, M., and Mazin, D. (2015). Ground-based detectors in very-high-energy gamma-ray astronomy. C. R. Phys. 16, 610-627. doi: 10.1016/j.crhy.2015.08.011

Elitzur, M., and Netzer, H. (2016). Disc outflows and high-luminosity true type 2 AGN. Mon. Not. R. Astron. Soc. 459, 585-594. doi: 10.1093/mnras/stw657

Elvis, M., Wilkes, B. J., McDowell, J. C., Green, R. F., Bechtold, J., Willner, S. P., et al. (1994). Atlas of quasar energy distributions. Astrophys. J. Suppl. 95, 1-68. doi: $10.1086 / 192093$

Harrison, C. (2014). Observational Constraints on the Influence of Active Galactic Nuclei on the Evolution of Galaxies. PhD Thesis, Durham University.

Kühr, H., Witzel, A., Pauliny-Toth, I. I. K., and Nauber, U. (1981). A catalogue of extragalactic radio sources having flux densities greater than $1 \mathrm{Jy}$ at $5 \mathrm{GHz}$. Astron. Astrophys. Suppl. Ser. 45, 367-430.

Lamastra, A., Menci, N., Fiore, F., Antonelli, L. A., Colafrancesco, S., Guetta, D., et al. (2017). Extragalactic gamma-ray background from AGN winds and starforming galaxies in cosmological galaxy formation models. Astron. Astrophys. 607:A18. doi: 10.1051/0004-6361/201731452

López-Gonzaga, N., Burtscher, L., Tristram, K. R. W., Meisenheimer, K., and Schartmann, M. (2016). Mid-infrared interferometry of 23 AGN tori: on

\section{AUTHOR CONTRIBUTIONS}

The author confirms being the sole contributor of this work and approved it for publication.

\section{ACKNOWLEDGMENTS}

Many of the concepts discussed here come from Padovani et al. (2017); I thank the co-authors of that paper for their work over many months in writing the review. the significance of polar-elongated emission. Astron. Astrophys. 591:A47. doi: 10.1051/0004-6361/201527590

Luo, B., Brandt, W. N., Xue, Y. Q., Lehmer, B., Alexander, D. M., Bauer, F. E., et al. (2017). The chandra deep field-south survey: 7 Ms source catalogs. Astrophys. J. Suppl. 228:2. doi: 10.3847/1538-4365/228/1/2

Padovani, P. (2016). The faint radio sky: radio astronomy becomes mainstream. Astron. Astrophys. Rev. 24:13. doi: 10.1007/s00159-016-0098-6

Padovani, P. (2017). On the two main classes of active galactic nuclei. Nat. Astron. 1, 0194. doi: 10.1038/s41550-017-0194

Padovani, P., Alexander, D. M., Assef, R. J., De Marco, B., Giommi, P., Hickox, R. C., et al. (2017). Active galactic nuclei: what's in a name? Astron. Astrophys. Rev. 25, 2. doi: 10.1007/s00159-017-0102-9

Padovani, P., Resconi, E., Giommi, P., Arsioli, B., and Chang, Y. L. (2016). Extreme blazars as counterparts of IceCube astrophysical neutrinos. Mon. Not. R. Astron. Soc. 457, 3582-3592. doi: 10.1093/mnras/stw228

Resconi, E., Coenders, S., Padovani, P., Giommi, P., and Caccianiga, L. (2017). Connecting blazars with ultrahigh-energy cosmic rays and astrophysical neutrinos. Mon. Not. R. Astron. Soc. 468, 597-606. doi: 10.1093/mnras/stx498

Richards, G. T., Lacy, M., Storrie-Lombardi, L. J., Hall, P. B., Gallagher, S. C., Hines, D. C., et al. (2006). Spectral energy distributions and multiwavelength selection of Type 1 Quasars. Astrophys. J. Suppl. 166, 470-497. doi: 10.1086/506525

Tavani, M., Barbiellini, G., Argan, A., Boffelli, F., Bulgarelli, A., Caraveo, P., et al. (2009). The AGILE mission. Astron. Astrophys. 502, 995-1013. doi: 10.1051/0004-6361/200810527

Urry, C. M., and Padovani, P. (1995). Unified Schemes for Radio-Loud Active Galactic Nuclei. Publ. Astron. Soc. Pac. 107:803. doi: 10.1086/133630

Wang, X., and Loeb, A. (2016). Contribution of quasar-driven outflows to the extragalactic gamma-ray background. Nat. Phys. 12, 1116-1118. doi: $10.1038 /$ nphys 3837

Conflict of Interest Statement: The author declares that the research was conducted in the absence of any commercial or financial relationships that could be construed as a potential conflict of interest.

Copyright (c) 2017 Padovani. This is an open-access article distributed under the terms of the Creative Commons Attribution License (CC BY). The use, distribution or reproduction in other forums is permitted, provided the original author(s) or licensor are credited and that the original publication in this journal is cited, in accordance with accepted academic practice. No use, distribution or reproduction is permitted which does not comply with these terms. 